\title{
Dor pélvica e achados indiretos da endometriose na ecografia pélvica: Uma
}

\section{correlação estatística}

\author{
Pelvic pain and indirect findlings of endometriosis on pelvic ultrasound: A statistical correlation \\ Dolor pélvico y hallazgos indirectos de endometriosis en la ecografía pélvica: Una correlación estadística
}

Recebido: 29/06/2021 | Revisado: 05/07/2021 | Aceito: 06/07/2021 | Publicado: 16/07/2021

\author{
Izabele Ferreira Pontes \\ ORCID: https://orcid.org/0000-0003-0694-4013 \\ Centro Universitário de Patos, Brasil \\ E-mail: belliipontes@gmail.com \\ Emerson Lopes Claudino \\ ORCID: https://orcid.org/0000-0002-8258-2934 \\ Centro Universitário de Patos, Brasil \\ E-mail: emerson.claudino@hotmail.com
}

\begin{abstract}
Resumo
Objetivo: Identificar a prevalência de mulheres com dor pélvica, quantas têm achados que sugerem endometriose na USG e os achados de endometriose mais frequentes e estratificação da prevalência dessas alterações ecográficas pela faixa etária. Metodologia: Trata-se uma pesquisa documental e que avalia a prevalência de quantas mulheres com dor pélvica crônica tem achados indiretos de endometriose na ecografia, possuindo, dessa forma, uma abordagem quantitativa. Tendo como local de estudo uma clínica particular chamada Clínica Valle Imagem na cidade de Itaporanga - Paraíba, contemplando a população de 87 mulheres com dor pélvica. Os instrumentos de coletas foram dados de laudos e prontuários médicos dos exames de ultrassonografia das pacientes com dor pélvica. Resultados: Foi observado que as 87 mulheres que apresentavam sinais clínicos de endometriose/adenomiose como dor pélvica crônica, dispareunia e infertilidade, 79 apresentavam alterações na ecografia que sugeriam endometriose. Conclusão: Embora a USG não seja o melhor método de diagnóstico de endometriose, já que a RM e laparoscopia são as de primeira escolha, a USG é sim um método útil para avaliação de pacientes com dor pélvica crônica pela sua disponibilidade, baixo custo e ser um método inócuo e pouco invasivo.
\end{abstract}

Palavras-chave: Endometriose; Dor pélvica; Ultrassonografia.

\begin{abstract}
Objective: To identify the prevalence of women with pelvic pain, how many have findings suggestive of endometriosis on USG, and the most frequent endometriosis findings and stratification of the prevalence of these ultrasound changes by age group.. Methodology: This is a documentary research that assesses the prevalence of how many women with chronic pelvic pain have indirect findings of endometriosis on ultrasound, thus having a quantitative approach, having as place of study a private clinic called Clínica Valle Imagem in the city of Itaporanga Paraíba, comprising a population of 87 women with pelvic pain. The collection instruments were data from reports and medical records of ultrasound exams of patients with pelvic pain. Results: It was observed that of the 87 women who presented clinical signs of endometriosis/adenomyosis such as chronic pelvic pain, dyspareunia and infertility, 79 presented changes on ultrasound that suggested endometriosis. Conclusion: Although USG is not the best method to diagnose endometriosis, since MRI and laparoscopy are the first choice, USG is a useful method for evaluation of patients with chronic pelvic pain because of its availability, low cost, and because it is innocuous and minimally invasive.
\end{abstract}

Keywords: Endometriosis; Pelvic pain; Ultrasonography.

\section{Resumen}

Objetivo: Identificar la prevalencia de mujeres con dolor pélvico, cuántas tienen hallazgos que sugieren endometriosis en la ecografía y los hallazgos más frecuentes de endomotriosis y la estratificación de la prevalencia de estas alteraciones ecográficas por rango de edad. Metodología: Se trata de una investigación documental que evalúa la prevalencia de cuántas mujeres con dolor pélvico crónico tienen hallazgos indirectos de endometriosis en la ecografía, teniendo así un enfoque cuantitativo, teniendo como lugar de estudio una clínica privada llamada Clínica Valle Imagem en la ciudad de Itaporanga - Paraíba, comprendiendo una población de 87 mujeres con dolor pélvico. Los instrumentos de recogida fueron los datos de los informes y las historias clínicas de los exámenes ecográficos de las pacientes con dolor pélvico. Resultados: Se observó que de las 87 mujeres que presentaban signos clínicos de endometriosis/adenomiosis como dolor pélvico crónico, dispareunia e infertilidad, 79 presentaban cambios en la ecografía que sugerían endometriosis. Conclusión: Aunque la USG no es el mejor método de diagnóstico de 
endometriosis, ya que la IRM y la laparoscopia son las de primera elección, la USG es un método útil para la evaluación de pacientes con dolor crónico de piel por su disponibilidad, su bajo coste y por ser un método inofensivo y poco invasivo.

Palabras clave: Endometriosis; Dolor pélvico; Ecografía.

\section{Introdução}

A dor é definida como sensações desagradáveis e emocionais por conta de acometimentos teciduais reais ou potenciais. Para a dor pélvica ser crônica ela tem que ter uma duração no mínimo de seis meses com intensidade que interfere nas atividades diárias e habituais. A dor pélvica ela pode ser derivada de várias etiologias como uma interação de sistemas do organismo humano, entre eles ginecológico, neurológico, intestinal, urinário, musculoesquelético, endócrino e psicológico. Por isso, nem sempre a dor pélvica nas mulheres está correlacionada com doenças ginecológicas, pois doenças como hérnia abdominal, síndrome do intestino irritável, urolitíases, cistites, neoplasias, síndromes miofasciais, hérnia de disco são possíveis fatores que podem causar a dor pélvica. (Carneiro et al., 2019)

Segundo Luz, Vila, Deus e Conde (2016), a dor pélvica crônica, sendo uma das afecções crônicas mais comuns na vida feminina, afeta diretamente na qualidade de vida, nas relações sociais, na saúde mental, e na saúde física. É uma situação em que estabelece sofrimento, estresse, insônia, ansiedade, depressão e compromete a relação sexual das mulheres. Embora essa dor crônica seja bastante vivenciada na vida delas, poucos foram os estudos sobre a prevalência DPC no mundo e as consequências na qualidade de vida e saúde.

A dor pélvica crônica é uma das causas de morbidade e incapacidade de funções em mulheres que pode interferir nas atividades habituais e do dia a dia e leva-las a procurar serviço de saúde. Segundo o autor Rocha et al. (2020), mulheres em qualquer idade e com idade reprodutiva, 3,8\% e $12 \%$ respectivamente relatam de sensações dolorosas na região pélvica, e que $18 \%$ das mulheres se afastam do trabalho pelo menos $1 \mathrm{vez}$ ao ano devido a dor.

Pensa-se em endometriose de acordo com as manifestações clínicas, o exame físico e os exames de imagem. O diagnóstico é comprovado com a presença de lesões diretamente biopsiadas ou excisadas cirurgicamente. Para ser diagnosticada, demora cerca de até 12 anos de início dos sintomas, e quando não se faz o tratamento, há correlação com uma má qualidade de vida, sintomas psicológicos, incapacidade funcional e disfunção sexual. Na endometriose, o sintoma mais frequente é a DPC que pode ser associado à dispareunia profunda e/ou sintomas urinários e gastrointestinais. Além disso, a infertilidade sem outra causa, pode vir como outro sintoma da doença (Tomás \& Metello, 2019). Alguns pacientes com endometriose podem relatar além de sintomas dolorosas, outras síndromes como síndrome do intestino irritável, síndrome de bexiga dolorosa, dores de cabeça até fibromialgias. Afecções nos ligamentos do útero-sacral podem aumentar a sintomatologia da dispareunia e as aderências do fundo de saco tem sido associada com dismenorreia grave. (Moraes, Tim \& Assis, 2020)

$\mathrm{Na}$ etiologia da endometriose há muitos casos assintomáticos e quando vêm a ter sintomas eles são inespecíficos por conta de outras condições clínicas, o que dificulta no diagnóstico e tratamento. Quando o exame físico ginecológico não vem normal, pode apresentar nodulações no colo uterino ou no fundo de saco de Douglas, e na palpação vaginal encontrar nodulações que ficam no fundo de saco vaginal posterior ou no septo reto-vaginal. A laparoscopia pode ser uma boa escolha para o diagnóstico, pois através da biopsia pode identificar e confirmar o diagnóstico. Há também a ecografia endovaginal, que não requer cirurgia se tornando menos invasivo, embora seja operador dependente, mas é de primeira linha quando se desconfia de uma endometriose pélvica e profunda infiltrativa, porém para esse último a RM ainda é de primeira linha por evidencia uma imagem mais clara a maioria das lesões (Tomás \& Metello, 2019).

Para uma abordagem na preservação qualidade de vida das mulheres com endometriose e na fertilidade, é importante que se faça um diagnóstico precoce, devido a doença ter caráter crônico e de ter possibilidade de avanço. (Vieira, Silva, Padilha \& Padilha, 2020). Para diagnóstico da endometriose são utilizados alguns exames de imagem como a ressonância 
magnética, a ultrassonografia e outro método definitivo, a laparoscopia. Embora, a RM seja um método de imagem para diagnosticar em casos de endometriose, a ultrassonografia pode ajudar a localizar lesões em útero, ovários e fundo de saco de Douglas. Além disso, baixo custo, bastante acessível, não expõe o paciente a radiação e avalia afecções pélvicas (Soares \& Costa, 2018)

$\mathrm{Na}$ maioria das localizações que tenha endometriose, a ecografia endovaginal apresenta maior especificidade e sensibilidade do que a RM. A American Society of Reproductive Medicina (ASRM), classificou de acordo com a avaliação cirúrgica a afecção em 4 estádios: a) Estadio 1: é mínima, os implantes endometriósicos são isolados e não possuem aderências; b) Estadio 2: é ligeira, os implantes são superficiais com menos de 5mm, possuem aderências ou são disseminados sobre a superfície dos ovários e peritônio.; c) Estadio 3: é moderado, os implantes são múltiplos superficiais ou invasivos, possuem aderências ao redor das trompas periováricas; d) Estadio 4: é grave, os implantes são múltiplos superficiais e profundos que apresentam grandes endometriomas ováricos e são frequentes aderências extensas. (Tomás \& Metello, 2019).

A ultrassonografia é um método que os seus achados são variados de acordo com o local da lesão da endometriose, sendo necessário antes de qualquer procedimento. É um exame que identifica endometriomas que são tumorações benignas, tendo como alcance de $96 \%$ de sensibilidade e $97 \%$ de especificidade nos diagnósticos e mapeamentos das afecções em locais de endometriose profunda da bexiga, intestino, no septo vaginal, ainda, ajuda na escolha do tipo de cirurgia. Sendo assim, a USG se mostra um exame de muita importância na prática ginecológica podendo avaliar a presença de endometriose e o comprometimento da pelve nos pacientes, além disso, é uma escolha de exame de imagem menos invasivo e com ótimo custobenefício. (Cruz, Amaral \& Amaral Filho, 2010)

Portanto, a ultrassonografia pode ser útil na avaliação da endometriose, principalmente em locais com baixa disponibilidade de exames de Ressonância magnética.

O objetivo desse estudo é identificar a prevalência de mulheres com dor pélvica, quantas têm achados que sugerem endometriose na USG e os achados de endometriose mais frequentes e estratificação da prevalência dessas alterações ecográficas pela faixa etária.

\section{Metodologia}

O estudo tem como condição metodológica uma pesquisa documental e que avalia a prevalência de quantas mulheres com dor pélvica crônica tem achados indiretos de endometriose na ecografia, possuindo, dessa forma, uma abordagem quantitativa. Esta forma de metodologia quantitativa, segundo Mussi, Mussi, Assunção e Nunes (2020) tem como principais características uma abordagem físico-numérica, onde não tem interesse ao individual, mas ao coletivo, além disso sua dinâmica da metodologia tem como potencialidade a análise de dados, consolidação da estatística, seleção das variáveis, nível de planejamento, seleção dos instrumentos que serão aplicados e da fundantação teórica. O local de estudo foi feito na cidade de Itaporanga - Paraíba, numa clínica particular chamada Clínica Valle Imagem, com a população de 87 mulheres com dor pélvica. A pesquisa foi anteriormente encaminhada ao Comitê de Ética do Centro Universitário de Patos - UNIFIP, assim foi obtido a aprovação.

Os prontuários das pacientes do estudo foram escolhidos de acordo com os critérios de inclusão, ou seja, as que tinham como principal sintomatologia dor pélvica e que fizeram USG transvaginal. Sendo assim, foi selecionado 79 pacientes. Os principais dados coletados foram: alterações sugestivas de endometriose, faixa etária das mulheres e alterações encontradas na ultrassonografia.

Os dados foram coletados através de laudos e prontuários médicos de exames de USG de pacientes com a dor pélvica da clínica particular. Através dos dados coletados, foram buscados os achados indiretos de endometriose. Após coletado os resultados obtidos no estudo, adiante foram analisados e comparados com pesquisas sobre o assunto através de artigos 
localizados em bancos de dados como o Scientific Eletronic Library Online (SCIELO), Literatura Latino-Americana e do Caribe em Ciências da Saúde (LILACS) e na plataforma Google Acadêmico e U. S. National Library of Medicine (PUBMED).

\section{Resultados}

Após coletado os dados dos prontuários que apresentavam sinais clínicos de endometriose/adenomiose referindo dor pélvica crônica, dispareunia e infertilidade, 79 delas apresentavam alterações na ecografia que sugeriam endometriose, o que significa 90,8\% apresentado no Gráfico 1 a seguir.

Gráfico 1 - Porcentagem das alterações sugestivas de endometriose.

\section{Porcentagem das alterações sugestivas de endometriose}

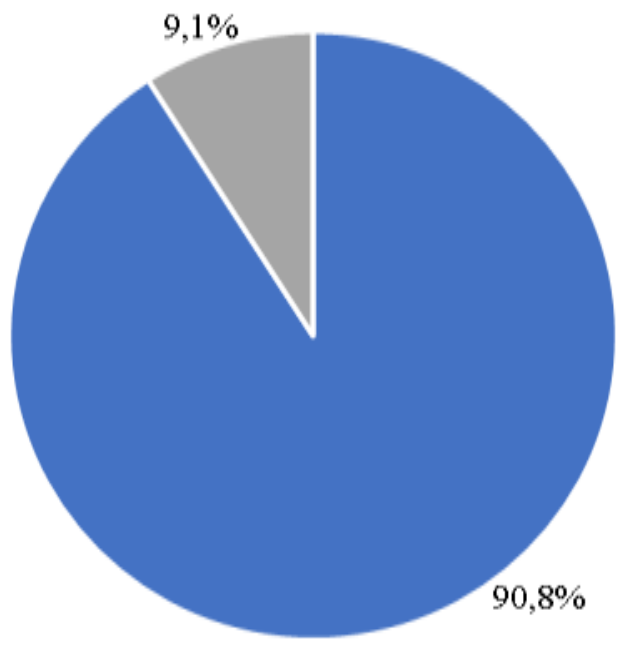

- Achados sugestivos de endometriose

- Sem achados sugestivos de endometriose

Fonte: Dados de pesquisa em prontuário (2021).

Os dados coletados no Gráfico 1 apresentam um total de 87 mulheres que demonstravam história clínica que sugeria endometriose como dor pélvica crônica, dispareunia e infertilidade, porém foi feito ultrassonografia nessas pacientes e, 79 delas $(90,8 \%)$ apresentavam achados sugestivos de endometriose e 9,1\% não apresentavam nenhum tipo de achado que sugeria a afecção.

Além disso, foi observado que as mulheres em estudo apresentam idades entre 10 a 50 anos, sendo que $45 \%$ delas se encontram em idade entre 30 a 40 anos, podendo ser visto no Gráfico 2. 
Gráfico 2 - Faixa etária das mulheres em estudo.

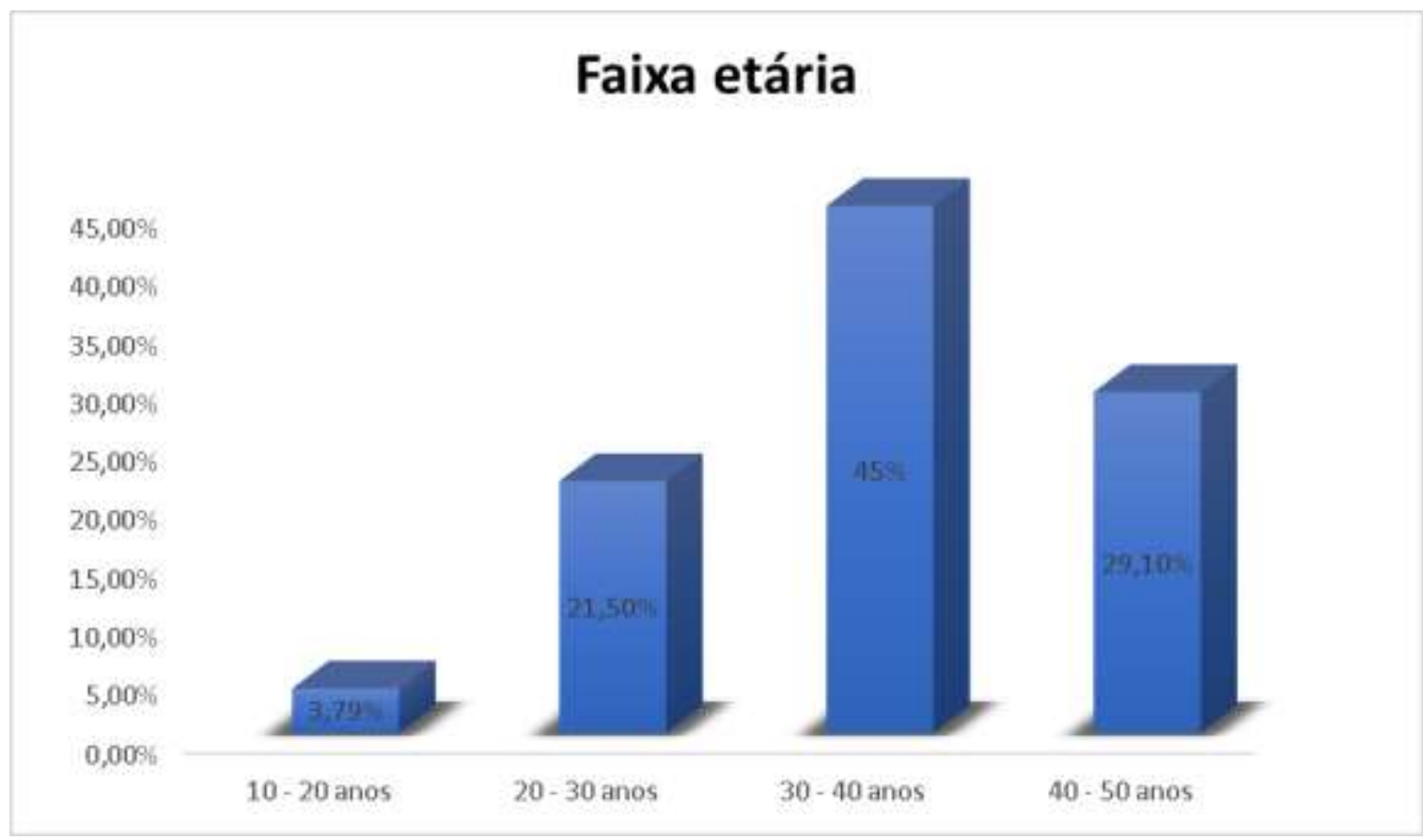

Fonte: Dados de pesquisa em prontuário (2021).

Através desses dados coletados no Gráfico 2, observa-se que foi comum achar alteração de endometriose em mulheres jovens adultas e com idades reprodutivas, mas que também a afecção pode aparecer em adolescentes e idades próximas ao climatério.

$\mathrm{Na}$ ultrassonografia realizada nas mulheres com dor pélvica, foi revelado as alterações sugestivas de endometriose/adenomiose evidenciado no Gráfico 3 a seguir.

Gráfico 3 - Percentuais das principais alterações encontradas na ultrassonografia.

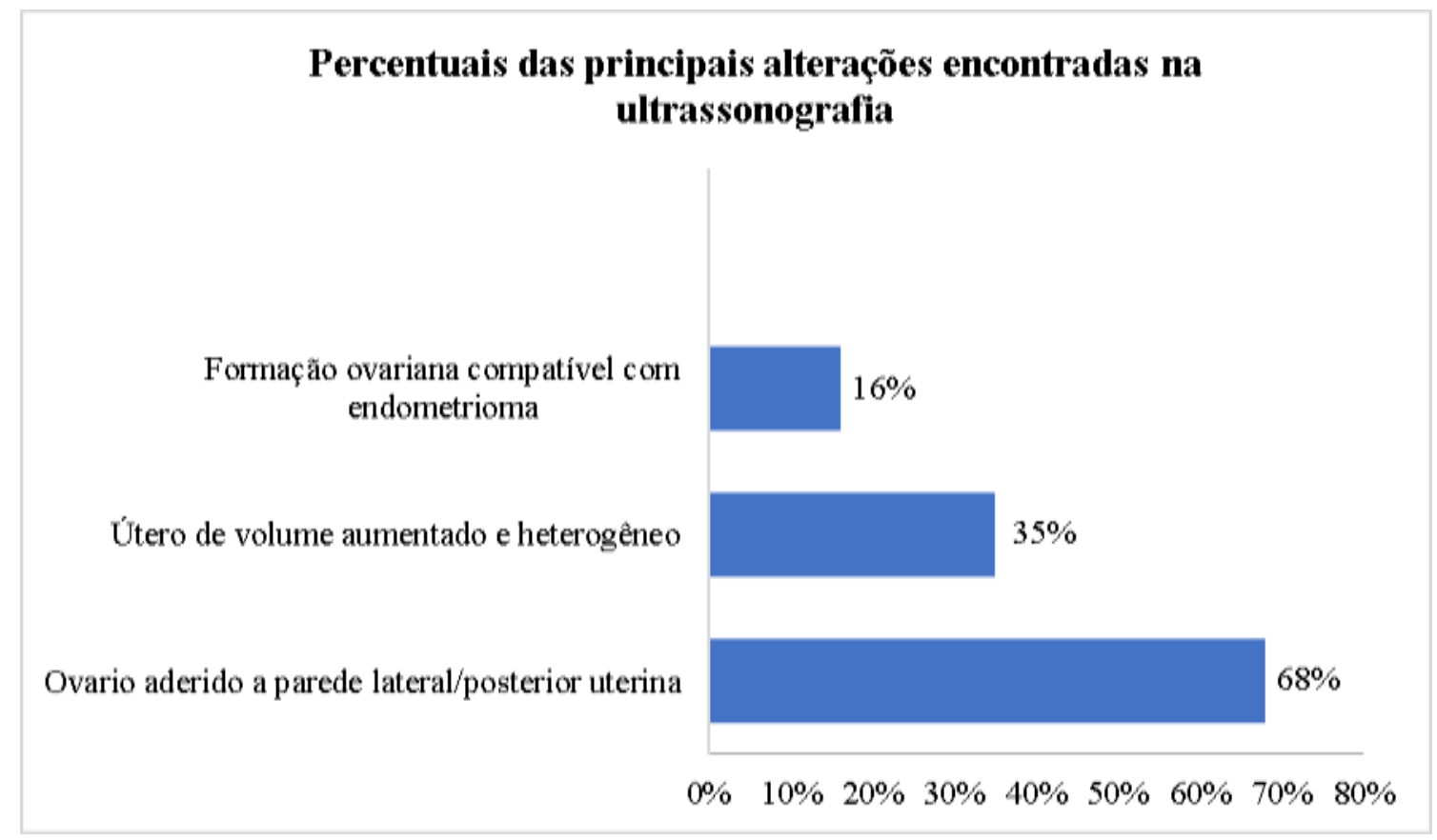

Fonte: Dados de pesquisa em prontuário (2021). 
As principais alteração observadas na ultrassonografia (representada no gráfico anterior) das 79 mulheres que apresentavam alteração de endometriose foram formação ovariana compatível com endometrioma, útero de volume aumentado e heterogêneo e ovariano aderido a parede lateral/posterior uterina. No Gráfico 3 apresenta o percentual de cada afecção onde se destaca o ovário aderido a parede lateral/posterior uterina em 53 mulheres correspondendo $68 \%$ do total.

\section{Discussão}

$\mathrm{Na}$ endometriose pélvica, é importante que se faça um diagnóstico preciso para uma melhor estratégia de tratamento. Os principais exames de imagem utilizados para detecção da patologia são a ultrassonografia transvaginal (USGTVS) e a ressonância magnética (RM). O exame mais disponível e de baixo custo relativo é a TVS, que pode ser usado como método de primeira escolha, que apresenta imagens detalhadas e dinâmicas da estrutura pélvica com o menor desconforto possível para as pacientes (Habib et al., 2020)

Segundo Augusto (2019) em seu estudo feito com 90 pacientes com endometriose no estado de Ceará, o principal sintoma era dor pélvica crônica em baixo ventre apresentando em $83 \%$ nas mulheres, seguindo de dispareunia apresentando em $40 \%$, a infertilidade foi em $22 \%$ das pacientes, as demais possuíam outras manifestações clínicas. As 79 pacientes do atual estudo, tinham como principal sintomatologia também a dor pélvica, a dispareunia e a infertilidade.

As sintomatologias responsáveis por sugerir endometriose nas pacientes encontrado nesse presente estudo também foram semelhantes a uma pesquisa feito por Yela, Quagliato e Pinto (2020) avaliou-se 60 mulheres com endometriose profunda com idade média de 37,2, ocorrendo sintomas clínicos em 50\% apresentavam dismenorreia, 57\% dispareunia e metade delas tinham dor pélvica crônica, sendo que ainda nesse estudos as pacientes relatavam a interferência da patologia com a qualidade de vida que afetava nos aspectos emocionais, autoestima, disposição, bem-estar social, relação sexual, entre outros.

Diante dos resultados encontrados nessa pesquisa em estudo, as alterações de endometriose na ultrassonografia tiveram incidência de 90,8\% em relação ao total das 87 mulheres que tinha como sintomas clínicos dor pélvica crônica, dispareunia e infertilidade, sendo a maioria delas entre as idades 20 a 40 anos. Dados semelhantes aos encontrados por Clemente et al. (2018), que analisou 97 pacientes que tinham suspeita de endometriose e delas cerca de $98 \%$ apresentavam a patologia através da ultrassonografia transvaginal (USGTV). Além disso, esse mesmo estudo as idades com maior incidência foi entre 20 a 40 anos $(79,4 \%)$ e apenas $1 \%$ menor que 20 anos.

Foram incluídos em um estudo feito por Oliveira et al. (2018) 85 mulheres com sintomatologia de dismenorreia, dispareunia, dor pélvica crônica, infertilidade e sintomas cíclicos intestinais ou urinários, e foram submetidas a USGTV. Do total, 29,4\% manifestaram achados que sugeriam endometriose, dessas as principais lesões encontradas foram endometrioma (60\%), lesões nas regiões retossigmóide (52\%) e retrocervicais (28\%). Além disso, 36\% dessas mulheres foram submetidas a videolaparoscopia e todas foram confirmadas com endometriose.

Resultados encontrados em um estudo feito por Florentino, Pereira, Martins, Lopes e Arruma (2019) analisou 65 mulheres do Brasil que tinham vida sexual ativa em idade fértil, onde elas apresentavam sintomas clínicos de endometriose, 80,6\% relatavam dor acíclica, 79\% dismenorreia, 61,3\% dispareunia e de 48,4\% infertilidade. Esses dados são importantes pois é uma associação da influência negativa na qualidade de vida gerada pela dor e na vida sexual. Comparando com o presente estudo, as pacientes também apresentavam as mesmas sintomatologias clínicas.

A Ultrassonografia transvaginal (USGTV) está sendo de uma importância como ferramenta para mulheres que apresentam dor pélvica crônica e suspeita de endometriose, é um exame muito preciso para detectar achados endométricos no útero e ovários, mas estudos demonstram a confiabilidade da USG no diagnóstico de infiltração profunda pélvica, no fundo de saco de Douglas, além das estruturas anteriores como endometriose profundo infiltrativa (DIE) urinário e aderências utero- 
vesíricas. Na questão da gravidade o uso de marcadores de USG como endometriomas e imobilidade ovariano também pode auxiliar na avaliação. (Reid \& Condous, 2016).

Segundo Oliveria et al. (2019) endometrioma ovariano é o achado mais comum da endometriose na USG-TV, sendo marcador de endometriose profunda mais grave. No presente estudo foi identificado $16 \%$ das pacientes teriam formação ovariana compatível com endometrioma, não sendo o mais prevalente dos achados, porém ainda é importante referir que essa lesão possui importante significado.

O estudo feito por Clemente et al. (2018) relataram que o principal compartimento encontrado com endometriose foi pélvico com 97,9\%, principalmente em úteros, ovários, ligamentos uterossacros, tubas uterinas, ligamentos largos e redondos e fundo de saco de Douglas sendo os mais atingidos. Dessa forma é raro encontrar em estruturas extra-pélvicas.

A pesquisa feita por Nisenblat, Bossuyt, Farquhar, Johnson e Hull (2016) comparando a RM com a Ultrassonografia Transvaginal para detecção cirúrgica de endometriose, referiu que nenhum dos dois exames foi eficiente para identificar endometriose pélvica global com exatidão suficiente para que fossem sugeridas substituir a cirurgia. Além disso, a USGTV, na endometriose infiltrativa profunda poderia ser o exame clinico para encontrar locais anatômicos comparando se com a ressonância magnética assim facilitando o planejamento do pré operatório.

Uma revisão sistemática feita por Moura et al. (2019), referiram que no diagnóstico de endometriose retossigmóide profunda a sensibilidade para USGTV foi de $73,3 \%$ a $98,1 \%$ e para RM 73,3\% a 100\%, ou seja, são valores semelhantes na precisão do diagnóstico. Dessa forma, a USG para ser usado como método inicial, mas se houver dúvidas a ressonância também é de escolha.

Uma comparação feita por Tomiyoshi et al. (2020) entre dois exames de imagem, analisou 63 mulheres que tinham suspeita de endometriose profunda e que poderiam ter comprometimento intestinal, $31 \%$ das pacientes apresentavam lesão até a gordura perirretal identificados pela USG e a RM 19\% delas. Já comparando as lesões que invadiram a muscular própria, 44\% foi diagnosticado pela USG 3D e 47\% pela ressonância magnética.

Em um estudo feito por Carmo (2016) comparou USG e RM em detecção da endometriose e conclui que ambos os métodos existem diferenças na visualização dos nódulos em ligamentos uterossacro e não detectavam bem o reto $\mathrm{e}$ retossigmóide. Já a USG tem melhor visão em estruturas superiores, superando a ressonância magnética no colón sigmoide, porém nos compartimentos posteriores, ela conseguiu se destacar, principalmente no septo retovaginal local mal visualizado na USG.

Os exames de imagem Ressonância magnética, USGTV e ultrassonografia transretal (TRUS) são métodos que podem detectar endometriose, um estudo feito por Zhang, He e Shen (2020) comparou os 3 métodos com um total de 30 estudos com 4565 participantes, a pesquisa obteve resultados precisos onde todos os métodos tiveram boa especificidade e sensibilidade para DIE, porém a USTV foi a única que preencheu o SpPIN que é positivo especifica nos critérios de triagem, com 95\% de especificidade. E para o diagnóstico de DIE, o TRUS foi o mais sensível. Ainda no estudo, os autores relataram que por mais que a USTV seja a modalidade de primeira linha após exame físico e história clínica, não substitui biópsia e laparoscopia que segue sendo o padrão ouro.

A Ultrassonografia tem uma significância na detecção não só em achados típicos de endometriose, mas também dos atípicos. Guerriero et al. (2020) fez um estudo sobre achados atípicos de endometriose e foi possível visualizar essas lesões em vários compartimentos como endometriose cicatricial, umbilical, do músculo reto, inguinal, apendicular, hepática superficial, pâncreas, rim e diafragma. $\mathrm{O}$ estudo também citou que por mais que esse exame de imagem seja importante para achados não típicos de endometriose, a RM ainda deve ser utilizada em alguns casos, pois para fazer diagnóstico diferencial é desafiador ou também USG não avalia todos os tipos de lesões endometriais. 
Um estudo feito por Rocha et al. (2020), com mulheres 25 mulheres até 44 anos de idade, teve como resultado que os níveis encontrados de incapacidade funcional eram relacionados a fatores como medo, preocupações e relatos sem conhecimento sobre a causa dor pélvica. As que tinha menor escolaridade relataram que as dores eram mais intensas, apresentavam mais preocupações, mais sofrimentos e maior incapacidade funcional. Além disso, as maiores médias de comprometimento psicológico eram ansiedade e estresse.

Há várias terapêuticas para o tratamento da endometriose, sendo eles a terapêutica médica com fármacos, cirurgia e atitude expectante. É necessário ter atenção no objetivo terapêutico, pois o tratamento é individualizado e depende da vontade do doente. Na terapêutica médica é fundamento o alívio da dor, utilizando analgésicos comuns, AINES e opióides, além disso pode diminuir a progressão da afecção utilizando medicação hormonal, porém o tratamento hormonal possui lado negativo quando ao tema de infertilidade pois alguns desses fármacos inibem a ovulação. O uso de hormônios, por exemplo a progesterona, pode aliviar sintomas como a dor pélvica crônica e dismenorreia, também tem a opção dos contraceptivos combinados, esses medicamentos são para controle da lesão e não a "cura". Na terapêutica cirúrgica, como todas as outros modelos de tratamento, é para a diminuição da dor, a recorrência e a fertilidade. (Tomás \& Metello, 2019)

A cirurgia pode diminuir os sintomas, porém após anos da cirurgia a doença pode voltar em até $50 \%$ dos casos. Algumas mulheres se tornam inférteis por conta da endometriose grave e progressiva, nesses casos há meios que ajudam a gravidez acontecer, como técnicas como a Fertilização In Vitro, a procriação médica assistida, entre outros. Há casos em que na endometriose a cirurgia não tem bons resultados em outcomes reprodutivos e dessa forma é de preferência que sejam encaminhadas para centros de procriação médica assistida do que ser submetidas a histerectomia. Nesse caso, a intervenção cirúrgica só é indicada quando é suspeito de malignidade em que a lesão tem um crescimento acelerado e são encontradas lesão atípicas no exame de imagem. Também é indicado a cirurgia quando a intervenção farmacológica não tem resultado significante e os sintomas são refratários, além disso estenose ureteral e sintomas intestinais pode ser indicado precocemente. (Tomás \& Metello, 2019)

\section{Conclusão}

De acordo com os resultados desse estudo, principalmente sobre os achados ecográficos, foi possível observar que 90,8\% das pacientes que possuíam sintomas clínicos como dispareunia, dor pélvica crônica e infertilidade, a ultrassonografia foi de fundamental importância para detectar lesões que sugerissem o diagnóstico de endometriose. Além disso, vários estudos citados na discussão apontam a alta especificidade e sensibilidade da USG para a detecção da patologia.

Sendo assim, levantando o questionamento se a USG não seria um exame de primeira escolha ao invés da RM, a USG é sim um método útil para avaliação de pacientes com dor pélvica crônica, pois através de um bom exame físico e história clínica, ela pode vir a ser a melhor metodologia para diagnóstico de endometriose pela sua disponibilidade, baixo custo e ser um método inócuo e pouco invasivo.

Levando em consideração que a ultrassonografia é um exame de imagem para diagnóstico de endometriose e que apresenta baixo custo e mais acessibilidade, é preciso mais estudos sobre esse método e demonstrar sua importância na detecção da afecção de endometriose, para que mais seja utilizado e pessoas de pouco poder aquisitivo tenham mais segurança na utilização desse exame.

\section{Referências}

Augusto, K. L. (2019). Manifestações clínicas relacionadas a endometriose em pacientes de um serviço de referência do estado do ceará. Journal of Coloproctology, 39(1), 147-148.

Carmo, C. O. D. (2016). Ressonância Magnética e Ultrassonografia Transvaginal na endometriose profunda: revisão sistemática. 
Carneiro, E. C. S. P., Silva, R. M. C. R. A., Pereira, E. R., Vallois, E. C., Chicaro S. C. R., \& Lima, M. M. S. (2019). Dor pélvica crônica feminina em relato de experiência: o singular norteando condutas. Nursing (Säo Paulo), 22(253), 2995-2998.

Clemente, A. G. M., Laureano, L. G., Yano, J. P. P., Evangelista, P. G., Amaral, W. N., Batista, L. A. A., \& Silva, W. R. (2018). Ultrassonografia transvaginal com preparo intestinal em pacientes com suspeita clínica de endometriose. Rev Bras de Ultrassonografia, 24, 7-10.

Cruz, R. C. L., Amaral, W. N., \& Amaral Filho, W. N. (2010). Diagnóstico ultrassonográfico da endometriose pelvica. Rev Goiana de Medicina, 39(1), 14-16.

Florentino, A. V. A., Pereira, A. M. G., Martins, J. A., Lopes, R. G. C., \& Arruma, R. M. (2019). Quality of Life Assessment by the Endometriosis Health Profile (EHP-30) Questionnaire Prior to Treatment for Ovarian Endometriosis in Brazilian Women. Rev. Bras. Ginecol. Obstet, 41(9), 548-554.

Guerriero, S., Conway, F., Pascual, M. A., Graupera, B., Ajossa, S., Neri, M., Musa, E., Pedrassani, M., \& Alcazar, J. L. (2020). Ultrasonography and Atypical Sites of Endometriosis. Diagnostics (Basel, Switzerland), 10(6), 345.

Habib, N., Centini, G., Lazzeri, L., Amoruso, N., Khoury, L. E., Zupi, E., \& Afors, K. (2020). Bowel Endometriosis: Current Perspectives on Diagnosis and Treatment. International journal of women's health, 12, 35-47.

Luz, R. A., Rodrigues, F. M., Vila, V. S. C., Deus, J. M., \& Conde, D. M. (2016). Qualidade de vida de mulheres com dor pélvica crônica. Rev. Eletr. Enferm, $17(3)$.

Morais, J. P., Tim, C. R., \& Assis, L. (2020). Considerations about the use of ozone therapy (O3) in the treatment of Endometriosis. Research, Society and Development, 9, (9), e403997616.

Moura, A. P. C., Ribeiro, H. S. A. A., Bernardo, W. M., Simões, R., Torres, U. S., D'Ippolito, G., Bazot, M., \& Ribeiro, P. A. A. G. (2019). Accuracy of transvaginal sonography versus magnetic resonance imaging in the diagnosis of rectosigmoid endometriosis: Systematic review and meta-analysis. PloS one, 14(4), e0214842.

Mussi, R. F. F., Mussi, L. M. P. T., Assunção, E. T. C., \& Nunes, C. P. (2020). Pesquisa Quantitativa e/ou Qualitativa: distanciamentos, aproximações e possibilidades. Revista Sustinere, 7(2), 414-430.

Nisenblat, V., Bossuyt, P. M. M., Farquhar, C., Johnson, N., \& Hull, M. L. (2016). "Modalidades de imagem para o diagnóstico não invasivo da endometriose." O banco de dados Cochrane de revisões sistemáticas, 2(2) CD009591.

Oliveira, J. G. A., Bonfada, V., Figueiró, M. F., Mungnol, T., Zanella, J. F. P., \& Coser, J. (2018). Clinical features, socio-demographic profile and ultrasound findings in women with endometriosis symptoms. Sci Med, 28 (4), ID30496.

Oliveira, J. G. A., Bonfada, V., Zanella, J. F. P., \& Coser, J. (2019). Ultrassonografia transvaginal na endometriose profunda: ensaio iconográfico. Radiol Bras, 52(5), 337-341.

Reid, S., \& George C. (2017). "Update on the ultrasound diagnosis of deep pelvic endometriosis." European journal of obstetrics, gynecology, and reproductive biology, 209, 50-54.

Rocha, J. N., Castro, L. E., Riccobene, V. M., Autran, M. S. M., Nogueira, L. A. C., \& Reis, F. J. J. (2020). Intensidade da dor, incapacidade funcional e fatores psicossociais em mulheres com dor pélvica crônica: um estudo transversal. BrJP, 3(3) ,239-244.

Soares, R. M., \& Costa, J. I. F. (2018). Achados ultrassonográficos da endometriose: principais apresentações e aspectos atípicos - ensaio iconográfico. Rev Med UFC, 58(4), 52-56.

Tomás, C., \& Metello, J. L. (2019). Endometriose e infertilidade-onde estamos? Acta Obstétrica e Ginecológica Portuguesa, 13(4), 235-241.

Tomiyoshi, M. M., Lima, D. M. R., Kurachi, G., Sagae, U. E., Emori, F., \& Bazzano, B. (2020). Correlation between findings of nuclear magnetic resonance and 3D anorectal ultrasound in patients with suspected deep endometriosis. J. Coloproctol, 40(3), 243-246.

Vieira, G. C. D., Silva, J. A. C., Padilha, R. T., \& Padilha, D. M. M. (2020). Endometriose: causas, implicações e tratamento da infertilidade feminina através das técnicas de reprodução assistida. Research, Society and Development, 9(10), e685910912.

Yela, D. A., Quagliato, I. P., \& Pinto, C. L. B. (2020). Quality of Life in Women with Deep Endometriosis: A Cross-Sectional Study. Rev. Bras. Ginecol. Obstet, 42(2), 90-95.

Zhang, X., He, T., \& Shen, W. (2020). Comparison of physical examination, ultrasound techniques and magnetic resonance imaging for the diagnosis of deep infiltrating endometriosis: A systematic review and meta-analysis of diagnostic accuracy studies. Experimental and therapeutic medicine, 20(4), 3208-3220. 